\title{
A Review on Structural Analysis of Human Retinal Vessels: Blood Vessels, Optic Nerve, Fovea Centralis and Related Diseases
}

\author{
Tooba Shafa ${ }^{1}$, Mohsin Jamil ${ }^{2}$ and Syed Omer Gilani ${ }^{1}$ \\ ${ }^{1}$ Department of Biomedical Engineering and Sciences \\ ${ }^{2}$ Department of Robotics and Artificial Intelligence \\ School of Mechanical and Manufacture Engineering (SMME), \\ National University of Sciences and Technology (NUST), \\ Islamabad Pakistan \\ tooba.shafa@yahoo.com,mohsin@smme.nust.edu.pk,omer@smme.nust.edu.pk
}

\begin{abstract}
This review describes different automated methods to locate the optic nerve in eye images. These tools are beneficial for eye specialists for the treatment evaluation and screening of patient and clinical study. These experiments done for the better results as first method by Adam Hoover et al. includes threshold probing to estimate the halting conditions of images with improvement of 15 times than previous work. Second work of Adam Hoover et al in 2003 describe method in which 31 images of retinas showing good condition and 50 images of retinas with disease were used, having such various symptoms as twisted vessels, choroid Neovascularization, and hemorrhages that make hard to define actual nerve, but this method achieved 89\% correct detection. Above all previous work most significant work done by the Alia Abdel Haleim et al in 2008, they done fuzzy convergence with illumination equalization and adaptive histogram equalization on retinal vessel images, these images were segmented by Gaussian matched filter. The proposed technique was assessed by STARE project's dataset, which contain 81 fundus images of both normal and diseased retinas, the OD center was detected correctly in 80 out of the 81 images (success rate of 98.77\%). Automatic diagnosis of blood vessels in the retina will help medical practitioner in detection of different eye diseases.
\end{abstract}

Keywords: Image processing, optic nerve, matched filters, thresholding, segmentation

\section{Introduction}

The optic nerve is a standout amongst the majority of essential organs in the human retina, the focal retinal course and focal retinal vein spread across cranial nerve, supply the blood to the upper covering of the retina [1]. Retina includes photoreceptors that are responsible for light processing named rods and cones. Rods are more sensitive than the cones and are responsible for the vision at low level of intensities as compared to these cones (mostly located in fovea centralis) are able to detect color with vision as they work at higher intensities. The optic nerve can be examined by the retinal fundus images; this information will be useful for treatment assessment of patient [13]. Vein appearance is an essential pointer for some judgments; including diabetes, hypertension, and arteriosclerosis additionally veins and courses have numerous discernible components, including distance across, shading, tortuosity and obscurity [10]. An exact depiction of the limits of veins makes estimations of these components possible. In this audit we depict a mechanized technique for various attempts to find and diagram veins in pictures

Received (August 28, 2017), Review Result (September 26, 2017), Accepted (October 5, 2017) 
of visual fundus and through this instrument, eye specialists can conceivably screen bigger populaces for vessel irregularities [1]. Vessels might be described by the normal shading, shape, angle and differentiation. For appropriate scopes of these traits, other picture expressions, for example, the limits of optic nerve and a few hemorrhages and sores, can show an indistinguishable neighborhood qualities from vessels [1].

Distinctive novel strategy was proposed in various period to fragment veins which compliments nearby vessel qualities with locale based characteristics of the system anatomy. A bit of vein system was estimated by testing a region of the MFR picture, redundantly diminishing the edge. At every cycle, locale based qualities of the piece are tried to contemplate test continuation and eventually to choose if that point is vessel. Pixels from tests that are not ordered as vessel are reused for additional probing. The quality of this perspective is that particular pixel names are guaranteed utilizing nearby and area based properties.

After probing method the improvise method was established for vessel segmentation that is fuzzy convergence in which different vessel divisions of a similar picture keeping in mind the end goal to strengthen the recognition of focalized focuses should be possible [14]. The thought was that the joining ought to be noticeable utilizing vessel divisions at various scales. The contribution of the calculation is a parallel division of the veins in which every vessel is demonstrated by a fluffy fragment. The yield from the calculation is a union picture, which is limit to recognize the most grounded purposes of union [2]. For more precise/ accurate results illumination equalization and histogram equalization was used [3]. Recent work done to localize the optic disc through correlation followed by vessel mask [16] and also by fractal method using eye structure and geometry [17].

\section{Methods}

Past techniques to portion veins for the most part fall into three classifications: (1) window based (2) classifier based and (3) tracking-based. Window based techniques, for example, edge location, appraise a match at every pixel for a given model against the pixel's encompassing window, the cross area of a vessel in a retinal picture was displayed by a Gaussian formed bend and after that identified utilizing turned coordinated channels. A comparable strategy was utilized for course identification in angiograms. The downside of these strategies was that the huge scale properties of vessels essentially disregarded to guarantee computational attainability. Classifier based techniques continue in two stages. Initial, a low-level calculation creates a division of spatially associated districts then these competitor locales are named being vessel or not vessel [4]. Tracking based techniques use a description guide to gradually venture along and portion a vessel. Vessel following continues from the papilla, stopping when the reaction to a one-dimensional matched filter falls beneath a given limit.

New technique was proposed for fragmenting veins in a retinal picture. The MFR picture is threshold utilizing a novel testing system. Picture was inspected by the test in pieces, testing various district based properties [Figure 1]. In the event that the test chooses that the piece is vessel, at that point the constituent pixels are all the while fragmented and characterized. When contrasted with classifier based strategies, this examining technique enables a pixel to be tried in various area designs before definite arrangement. Correlation with tracking based strategies, examining strategy was driven by a two-dimensional MFR. Testing strategy is locale based thus normally takes into account various branches. Right off the bat, the coordinated channel development and convolution was done, whereupon calculation manufactures, at that point limit testing and its application to vein division in a retinal picture. 


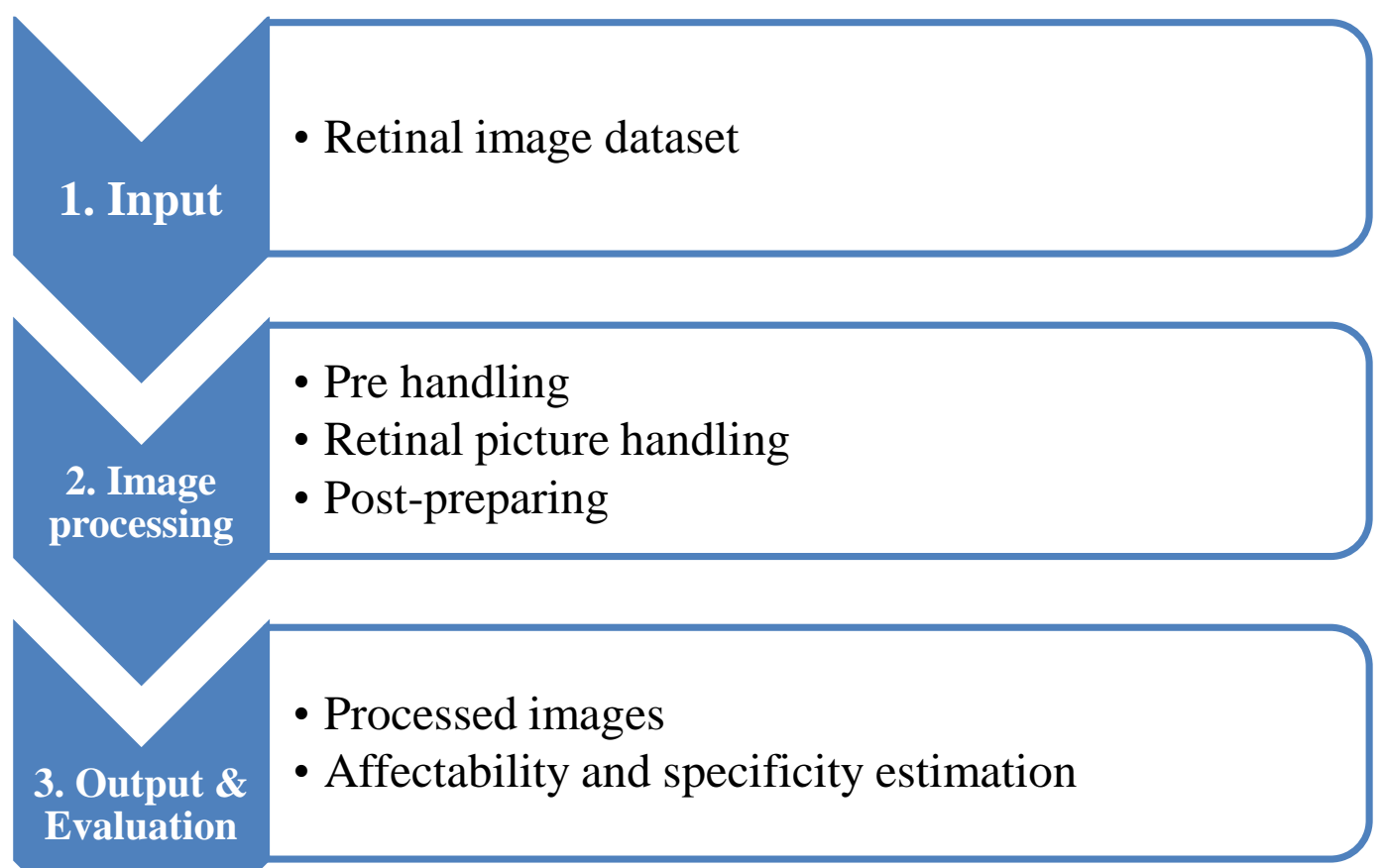

Figure 1. Image Processing [9]

\subsection{Matched Filter for Blood Vessels}

The coordinated channel depicts the normal appearance of a coveted flag, for motivations behind relative coordinating. Gaussian capacity was presented as a guide for a vein description. The model was reached out to two measurements by expecting that vessel has a settled width also bearing a short length. Channels were actualized utilizing twelve 16 x 16 pixel pieces. A coordinated channel was connected a retinal picture with twelve bits by convolution. The MFR was considering as the incentive for the most noteworthy scoring portion at every pixel. For the reasons for edge testing, the MFR picture is standardized and quantized to eight bits for each pixel [1].

\subsection{Threshold Probing}

The essential operation of this calculation was to test districts in a MFR picture, amid each test, an arrangement of criteria is tried to decide the edge of the test and eventually to choose if the zone being examined/piece is vein [2]. Every pixel in the test was utilized as a beginning stage for edge examining this testing is iterative [11].

Past work has been done to fragment a retinal picture into supply routes, veins, the optic circle and to track eye developments in light of retinal elements, including the nerve, utilizing techniques split-and-union division, trailed by highlight based grouping, finding the brightest locale in picture and fundamental thresholding for splendid zones separately by Y. Tolias et al., in 1998.

In 2003 Hoover et al utilize vein union as the essential element for recognition. This strategy was tried on 81 pictures (31 pictures of healthy retinas and 50 pictures of sick retinas) demonstrating an assortment of retinal maladies, befuddling sores and appearances. On this informational collection, this technique accomplishes a 89\% right recognition rate. This technique distinguishes the optic nerve as the point of convergence of the vein arrange. 


\subsection{Fuzzy Convergence}

In fuzzy every line like form is demonstrated by a fuzzy portion and it union is a voting-based strategy. The aggregation of these votes at every pixel creates a picture guide where every pixel contains esteem proportionate to its quality of union. The guide is then obscured and edge to create at least one purposes of most grounded meeting [5].

\subsection{Illumination Equalization}

The light in a retinal picture was uneven; the irregularity is fundamentally because of an optical abnormality called vignetting. Vignetting is the consequence of a dishonorable centering of light through an optical framework, so as to fix this apply enlightenment evening out to the picture in [6]. To enhance the visual qualities of an image, like brightness, color and position, simultaneous contrast can be done which correct the luminance by gamma correction when one object/light patch in image wrongly affected by the other object/light patch and explain optical effects. Luminance value is from 0 (black) to 1 (white), gamma correction results in correction of these intensities when image seems too dark or bleached on the other hand it also have the effects for red, blue and green intensities [15].

\subsection{Localization of OD by Mask Generation, Illumination Equalization, AHE, Blood Vessel Segmentation and Matched Filter}

In [3] review done propelled by the work of Frank ter Haar, Hoover and Goldbaum and Foracchia et al., they proposed a more ad libbed technique for the programmed discovery of the OD. The exhibited strategy includes a few stages; at first, a binary mask is created, at that point the brightening and differentiation all through the picture are evened out lastly, the retinal vasculature was portioned and headings of the vessels were coordinated to the proposed channel which speaks to the normal vessels' bearings in the OD region.

Mask generation mark the pixels having a place with the round retinal fundus region of interest in the whole picture and furthermore reject the foundation of the picture from further calculations and handling. The technique utilized was proposed by Frank ter Haar, who connected an edge to the pictures and after that the morphological administrators were connected individually utilizing a $3 \times 3$ square part to give the last area of-intrigue cover.

The brightening of retinal was non-uniform because of the variety of the retina reaction and non-consistency of image framework. Vignetting or different types of irregular brightening make the run of the mill examination of retinal pictures illogical and pointless, the light leveling connected to pictures [3]. At that point versatile histogram adjustment connected to standardize and improve the complexity inside fundus pictures; this technique is more viable than the established histogram evening out, particularly when distinguishing little veins portrayed by low differentiation levels.

For retinal veins division, they utilized the basic and standard edge fitting calculation proposed by Chaudhuri et al., [1]. With a specific end goal to create a paired vessel/nonvessel picture, the most extreme reactions are edge utilizing the worldwide edge determination calculation. 12 formats connected to the adaptively histogram evened out picture fundamentally enhances the division calculation and builds the affectability and specificity of the distinguished vessels. The vessels direction map (VDM) can be acquired by division calculation through recording a heading of layout which accomplished most extreme reaction at every pixel.

A coordinated channel portrays the normal appearance of a coveted flag for reasons for relative displaying in [2]. In this way, with a specific end goal to distinguish the OD, a basic vessels' course coordinated channel was presented to generally coordinate the bearing of the vessels at the optical disk region. The 9x9 layout was resized by bilinear 
insertion to sizes $241 \times 81,361 \times 121,481 \times 161$, and $601 \times 201$ to coordinate the structure of the vessels at various scales. The distinction between every one of the four layouts and a VDM was computed and the pixel having the slightest collected contrast was chosen as the OD focus in [3].

In 2016 M. Jamshidi et al., done work on 100 (40 from DRIVE and 60 from other database) images to proposed a geometrical parametric model for the accurate detection of vessels. They firstly estimate the optic cup by its brightness that is more than other neighborhood regions. For this cup estimation they use two strategies known as correlation and least square. Correlation done to correlate the estimation with original gray scaled image. This estimated the position of cup accurately. After this vessel mask technique used to confirm the results of correlation for optic disc localization. Next estimation method was least square which used points of the estimation to compute the sum of squares of deviation of data points.

\section{Results}

\section{Table 1. OD Detection Results for the Proposed and Literature Reviewed Methods}

\begin{tabular}{|c|c|c|c|}
\hline Technique name & Features & Accuracy & Remarks \\
\hline $\begin{array}{l}\text { Adam Hoover and } \\
\text { Michael Goldbaum } \\
\text { (2003) }\end{array}$ & $\begin{array}{l}\text { Optic nerve detection by } \\
\text { fuzzy convergence of } \\
\text { blood vessels }\end{array}$ & $89.0 \%$ & $\begin{array}{l}\text { Accurately detect the } \\
\text { Neovascularization and } \\
\text { hemorrhages in diseased } \\
\text { retina by comparison to } \\
\text { healthy ones. }\end{array}$ \\
\hline $\begin{array}{l}\text { Frank ter Haar } \\
(\mathbf{2 0 0 5 )}\end{array}$ & $\begin{array}{l}\text { Fitting the vasculature } \\
\text { orientation on a } \\
\text { directional model }\end{array}$ & $93.8 \%$ & $\begin{array}{l}\text { Analysis of anatomical } \\
\text { structures of retina by the } \\
\text { detection of OD. }\end{array}$ \\
\hline $\begin{array}{l}\text { Aliaa Abdel-Haleim } \\
\text { Abdel-Razik } \\
\text { Youssif et al. (2008) }\end{array}$ & $\begin{array}{l}\text { Optic disc detection by } \\
\text { vessels' direction } \\
\text { matched filter }\end{array}$ & $98.8 \%$ & $\begin{array}{l}\text { Optic disc detection for } \\
\text { developing automated } \\
\text { assessment systems for } \\
\text { disease treatment. }\end{array}$ \\
\hline $\begin{array}{l}\text { Adam Hoover et al. } \\
\text { (2000) }\end{array}$ & $\begin{array}{l}\text { Threshold probing to } \\
\text { locate blood vessel }\end{array}$ & $\begin{array}{l}\text { Upto } \\
90 \%\end{array}$ & $\begin{array}{l}\text { Automated method to } \\
\text { locate blood vessel for } \\
\text { patient treatment and } \\
\text { clinical study. }\end{array}$ \\
\hline $\begin{array}{l}\text { M. Jamshidi et al } \\
\text { (2016) }\end{array}$ & $\begin{array}{lr}\text { Correlation, } & \text { least } \\
\text { square and } & \text { vessel } \\
\text { mask for } & \text { OD } \\
\text { localization } & \end{array}$ & $98.33 \%$ & $\begin{array}{l}\text { Simple mathematical } \\
\text { method that focused on } \\
\text { optic nerve head contour } \\
\text { that needed to locate OD. }\end{array}$ \\
\hline
\end{tabular}




\begin{tabular}{|l|l|l|l|}
\hline $\begin{array}{l}\text { Madhukar Bhat et al } \\
\mathbf{( 2 0 1 7 )}\end{array}$ & $\begin{array}{l}\text { Pixel based multi } \\
\text { fractal analysis to } \\
\text { detect OD }\end{array}$ & $98.66 \%$ & $\begin{array}{l}\text { Automated detection } \\
\text { method of OD by eye } \\
\text { structure with improvising } \\
\text { time complexity. }\end{array}$ \\
\hline
\end{tabular}

The MFR pictures were prepared utilizing fundamental thresholding for each of the 20 pictures [12]. Execution was built up as takes after; any pixel (hand named as vessel), MFR was likewise over the given limit, was considered a genuine positive and any pixel (hand named as non-vessel), MFR was additionally over the given edge, was considered a false positive [2]. The genuine positive rate was built up by separating the quantity of genuine positives by aggregate number of pixels hand marked as vessel; the false positive rate was set up by partitioning the quantity of false positives by the aggregate number of pixels hand named as non-vessel.

Then again in the work of Adam Hoover and Michael Goldbaum, they tried their strategy on 31 pictures of sound retinas and 50 pictures of retinas with ailment, the nerve was unmistakable in each of the 81 pictures, despite the fact that in 14 pictures it was on the fringe of the picture so that exclusive a small amount of the nerve was noticeable and in five of the pictures the nerve was totally darkened by draining [7]. Techniques were tried in four adjustments; (a) equalized brilliance utilizes illumination equalization taken after by speculation era, (b) fuzzy convergence to single vein division, (c) fuzzy convergence to 6 divisions of veins, each at an alternate scale, (d) fuzzy convergence and leveled splendor were joined. In the event that the outcome from fuzzy convergence was esteemed uncertain, at that point the outcome from balanced splendor will be utilized.

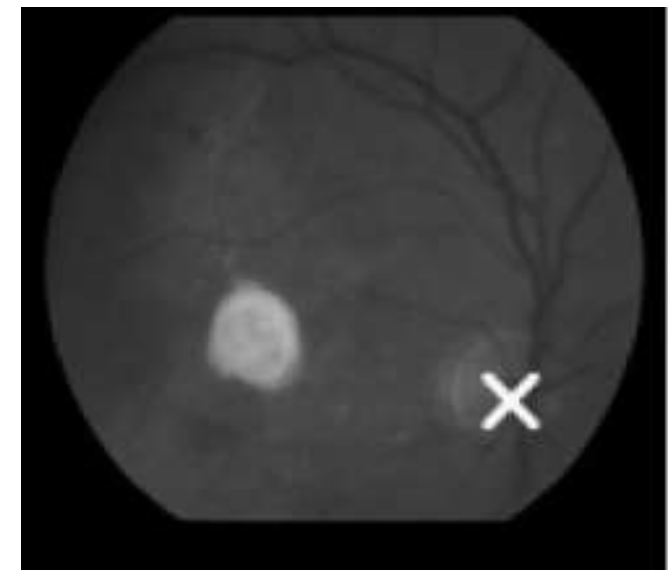

(a)

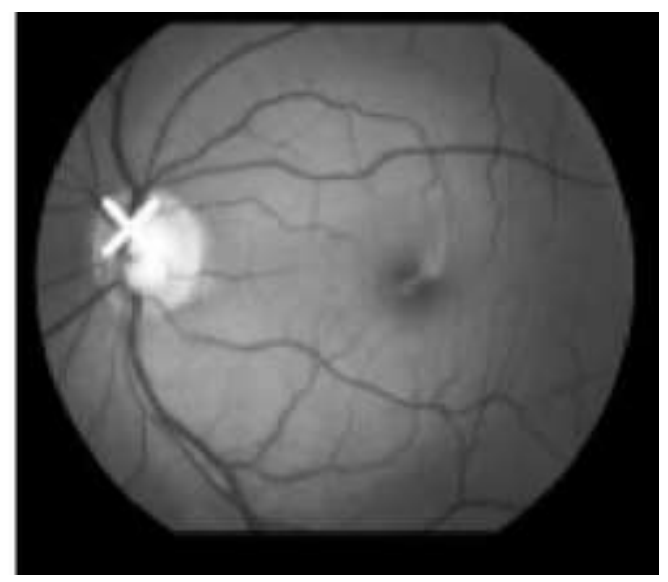

(b)

\section{Figure 2. Successful Results of Images by Adam Hoover and Michael Goldbaum Experimentation [8]}

Experimentation of Adam Hoover and Michael Goldbaum shows that the combination of two techniques fuzzy convergence and equalized brightness gives the highest accurate results for nerve detection [Figure 1]. They also notify the failure of their method that is due to the convergence of blood vessels around bright lesions.

In contrast with past performed techniques the proposed strategy for Aliaa AbdelHaleim et al., in 2008 made a progress rate of $98.77 \%$ [Table 1] The assessed OD focus was viewed as right if that was situated inside 60 pixels of the physically distinguished focus [8]. Normal separation between the assessed OD focus and the physically recognized focus was 26 pixels. Furthermore, the OD was recognized effectively in the 
greater part of the 40 DRIVE dataset pictures (a 100\% achievement rate) utilizing the proposed technique and the normal separation between the evaluated and the physically distinguished OD focuses were 17 pixels.

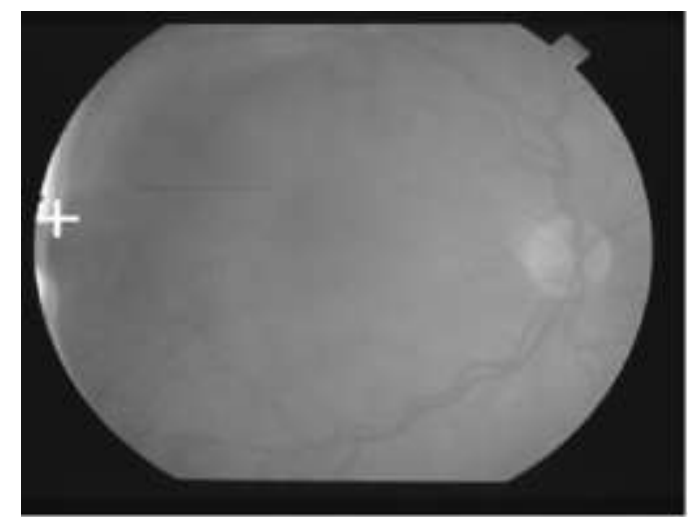

(a)

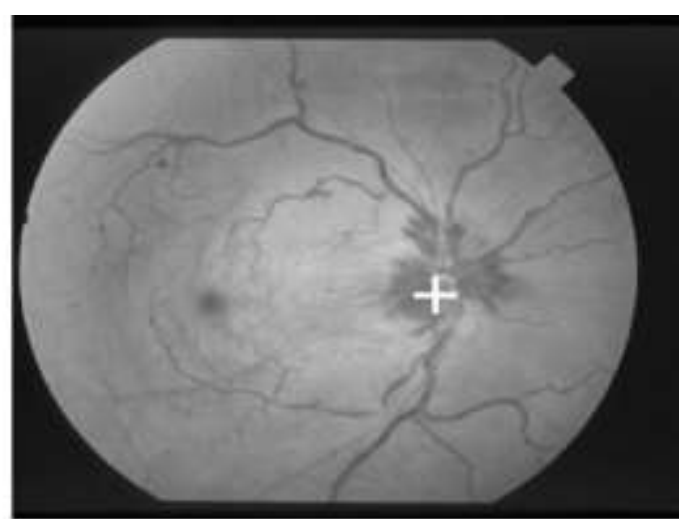

(b)

Figure 4. Results of Aliaa Abdel-Haleim et al. (2008) Work. (a). Failure Result (b). Accurate Detection of OD [3]

Aliaa Abdel-Haleim et al., work shows maximum accuracy in OD detection [Figure 4. (b)] but there is also present drawback of this technique that is incorrect detection of OD due to unequal semicircular appearance brilliance close to the edge that influenced the segmentation of vessels but that failure was noticed in 1 out of 81 [Figure 4. (a)].

M. Jamshidi et al., (2016) experiment shows accurate results of localization in 39/40 of DRIVE dataset and in all 60 images of other database with failure of 1 image only that could be resolve by vessel mask method. In Figure 1 left side image is the original image from DRIVE, middle one is the image that shows optic cup marked with a red point and on the right side there is an estimated image. 1 failure result was improvised by using vessel mask technique to detect the optical disk [Figure 3.1] and Figure 3.2 shows the circular image of optic disc using least square. Least square method also confirmed the reliability of correlation method results but correlation has better results than least square with vessel mask [16].
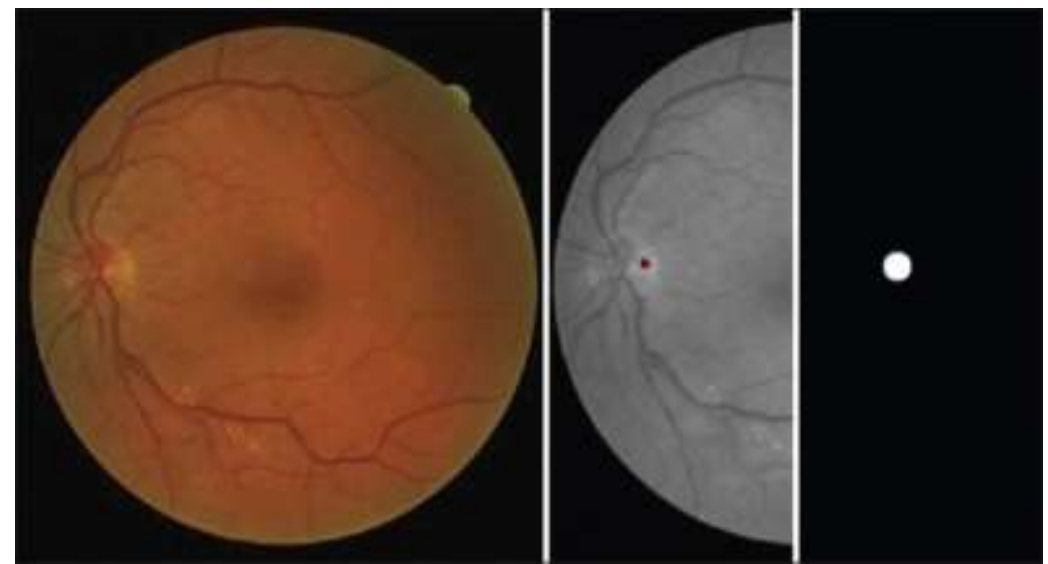

Figure 3. Detected Areas by Correlation Method [16] 

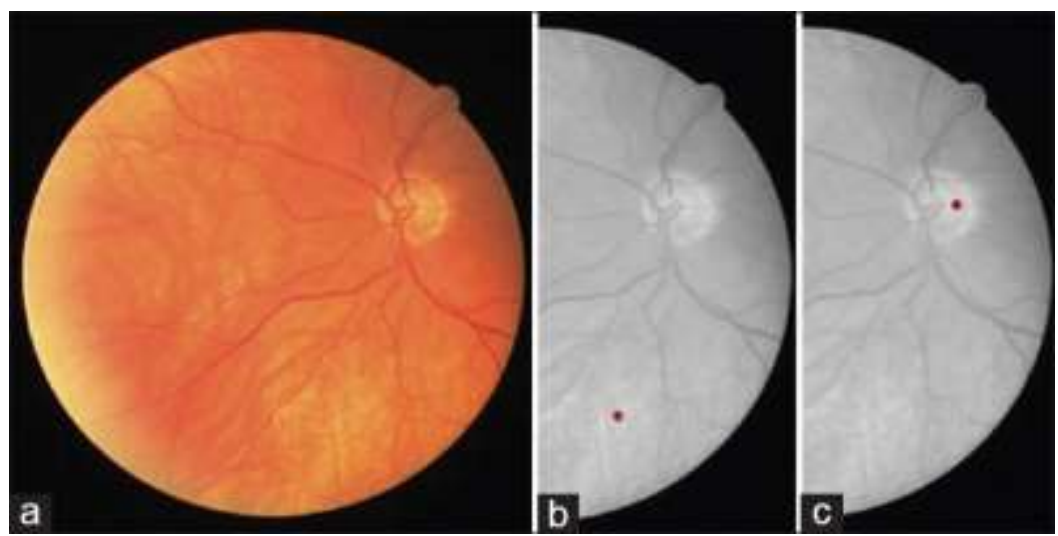

Figure 3.1. (a). DRIVE Dataset Image 23 (b). Failure of Correlation to Detect OD (c). Localization of OD by Additional Technique of Vessel Mask [16]

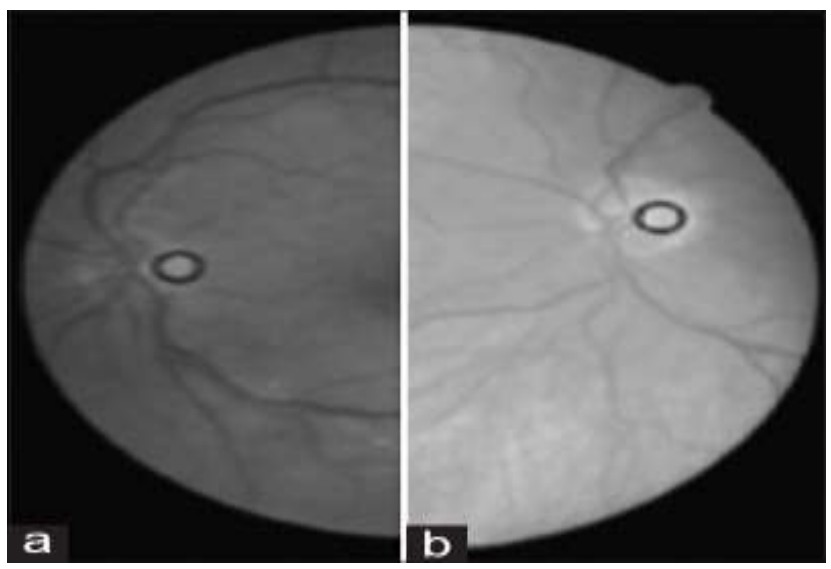

Figure 3.2. (a) Least Square on Image 1 of DRIVE (fig 3 Shows Original Image) (b) Least Square on Image 23 of DRIVE [16]

Recently Madhukar Bhat et al., (2017) work on the detection of optic disc by fractal analysis [Figure 5. (a), (b)

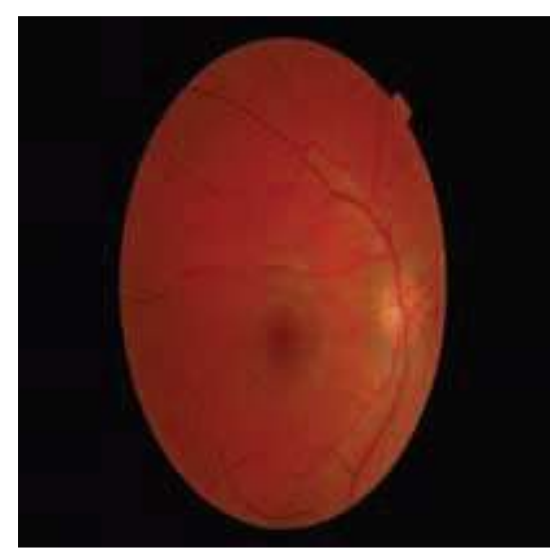

(a)

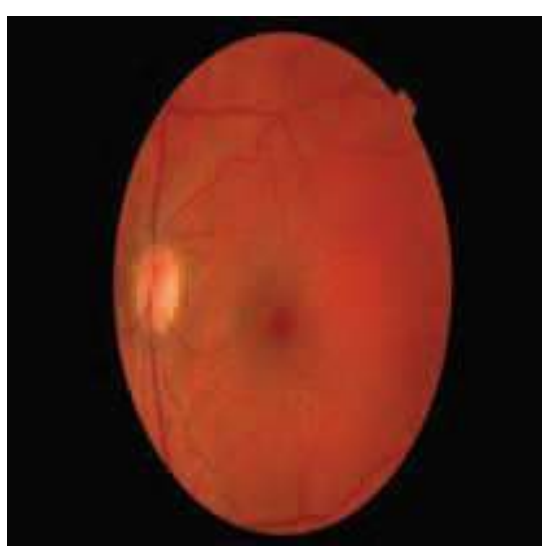

(b)

Figure 5. Original Images [17]

They performed Pixel Based Multi Fractal Analysis to explore the eye structure. Firstly, images of retina were pre-processed by modified method of contrast limit adaptive histogram equalization (CLAHE) [Figure 6], followed by mean based 
localization for localization of region of interest (ROI) [Figure 7] that was further processes to improve the developed algorithm's time complexity and efficiency [17].

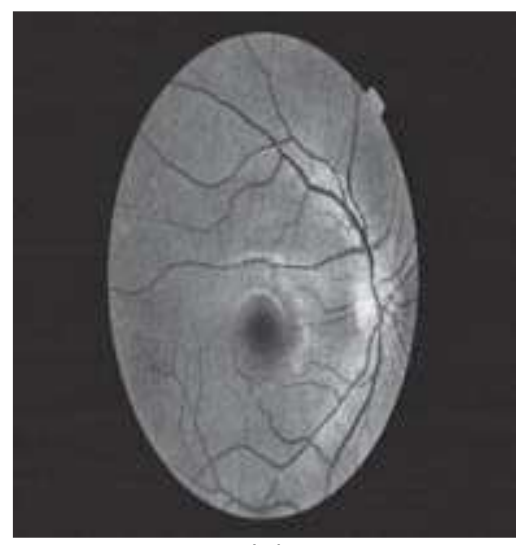

(a)

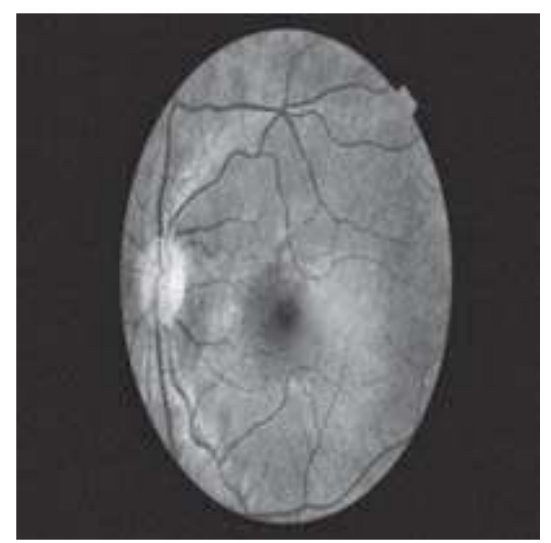

(b)

Figure 6. Pre-processed Images [17]

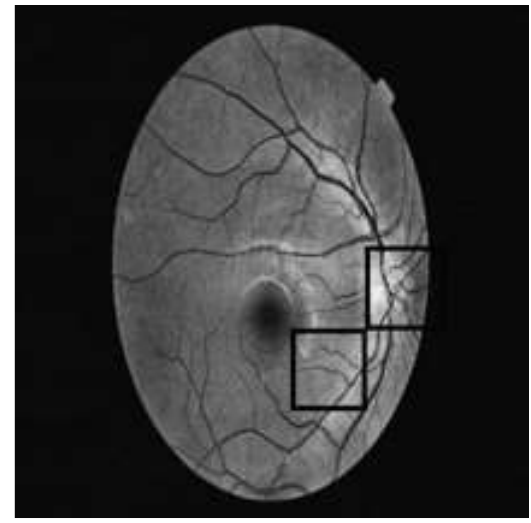

(a)

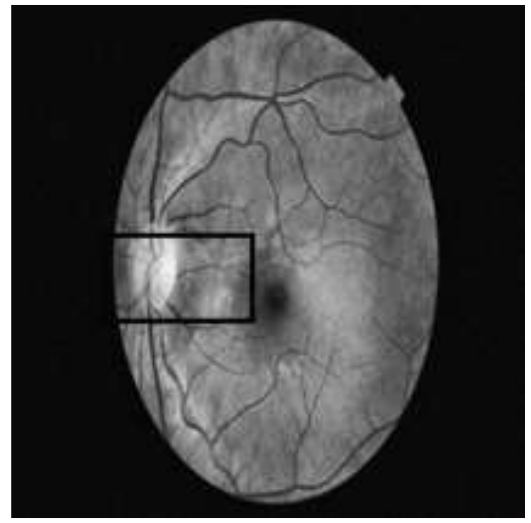

(b)

\section{Figure 7. Region of Interest by Mean based Localization [17]}

Located region of interest goes under the process of Canny Edge detection leveraged with Gabor operator that extracts blood vessels from the retinal image. Origin of vascular tree was located by pixel based multi fractal analysis, depend upon the fact that it has highest fractal dimension [17]. This gave the accurate location of centre followed by which OD of the retinal image was traced. The algorithm was done on 50 images (STARE, DRIVE and MESSIDOR) and gave $98.66 \%$ accurate results by this method [Figures 8, 9]. 


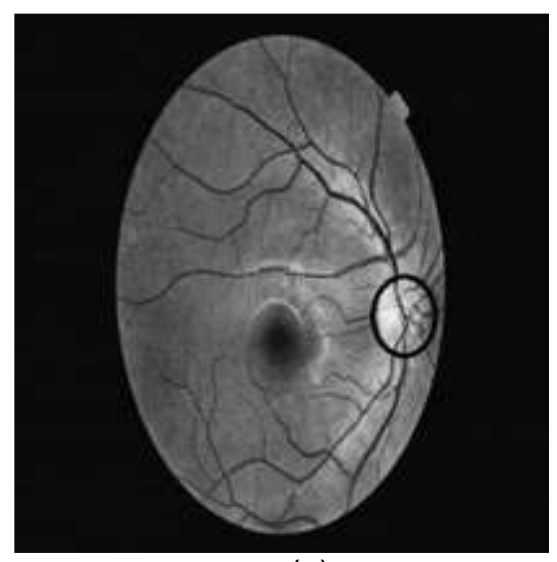

(a)

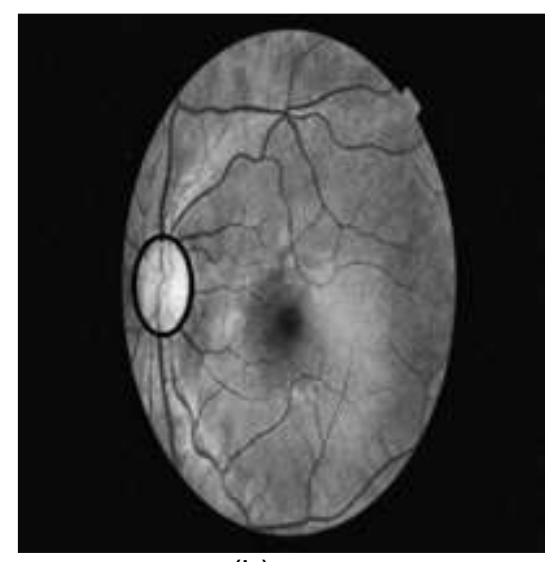

(b)

Figure 8. Traced Results of Optic Disc [17]

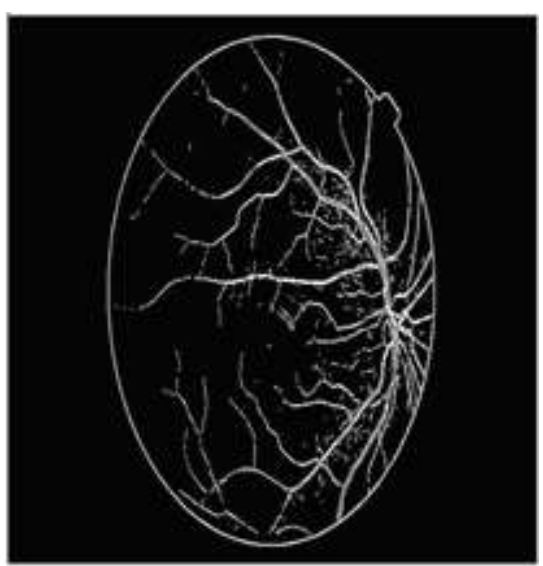

(a)

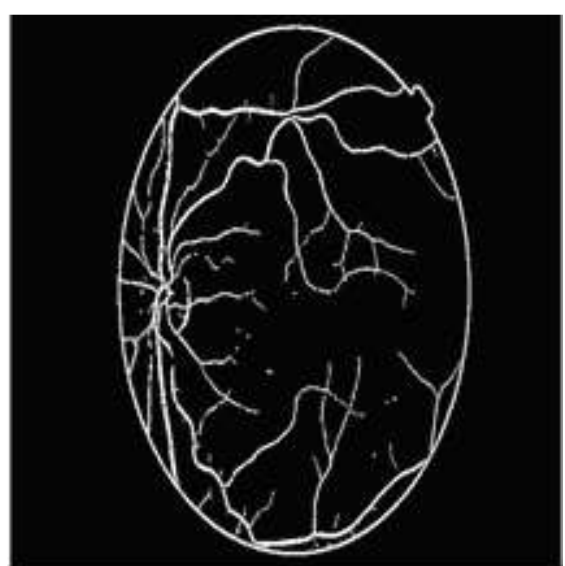

(b)

Figure 9. Results of Blood Vessel Segmentation [17]

\section{Conclusion \& Future Aspects}

Image processing is done to enhance the quality of an image, can be done by many ways including image classification, segmentation, feature extraction, enhancement and image acquisition use to remove noise and abnormalities. Occasionally image may be insufficient in brightness and contrast these problems solved by the contrast stretching, histogram modification (histogram equalization: use to enhance contrast by reconstruction/adjustment of pixels and image intensities) edge detection and sharpening. These processed images can be stored in various ways inclusively magnetic (floppy diskette, hard drive, magnetic strip tape cassettes), optical (CD-ROM disk, blueray disk, DVD) and flash memory devices (USB flash drive and memory card). These devices used for information holding for temporarily or permanently. Flash memory is the most efficient, cheap and reliable method these days to store data/information than others.

Different methods were investigated of iteratively tracking along blood vessels in a digitized image of the retina, recording information on the morphology of the individual vessels and their macrostructure but there is some problem that this is not easy to locate optical disc in the presence of any lesion. The work of Adam Hoover et al., (2000) clarifies that the threshold probing used to decide the ending conditions in retinal pictures. The use of this technique to 3D vessel division on information from other imaging modalities is additionally a theme of current intrigue. Thus, Adam Hoover et $a l .$, (2003) additionally find retinal veins by improvised strategy for convergence for 
more exactness; these techniques utilize the meeting of the vein arrange as the essential component for location, in conjunction with the brilliance of the nerve as an optional element. They effectively distinguish the nerve in $89 \%$ of the cases and in $100 \%$ of the healthy cases. There strategy for finding the union of the vein system depended on a novel calculation known as fluffy merging. In future fuzzy meeting may discover value in other medicinal applications other than optic nerve recognition.

As compared to these the work of Aliaa Abdel-Haleim et al., (2008) includes mask generation with illumination equalization and adaptive histogram equalization following by vessels' direction matched filter and blood vessel segmentation. This method attained $98.77 \%$ success rate to locate the OD. Illumination equalization normalized the glow over the fundus picture, expanding the quantity of OD hopefuls inside the OD district. Adaptive histogram equalization (AHE) changed the picture into more proper appearance for the utilization of the division calculation. The 2-D vessels' heading coordinated channel was fruitful in speaking to the leading representation of the retinal vessels encompassing the OD, proposed technique accomplished better outcomes contrasted with the investigated strategies.

Proposed method of Madhukar Bhat et al., (2017) was tested on 150 images from 3 databases and resulted in $98.66 \%$ accurately detection of OD. They emphasizes that this method is completely real time automated implemented on 150 retinal images for the detection of optical disc.

Considering the recent advances more adoption of retinal imaging can be expected in the clinical practice in the future for early identification of several chronic diseases and long term conditions. Increasing amount of retinal images indicates that the application of automatic image analysis techniques are expected to become more important to aid the work of medical experts and to decrease the associated health care costs. The automatic analysis of retinal images may also further encourages the establishment of large scale computer aided screening and prevention programs.

\section{References}

[1] S. Chaudhuri, S. Chatterjee, N. Katz, M. Nelson and M. Goldbaum, "Detection of blood vessels in retinal images using two-dimensional matched filters", IEEE Trans. Med. Imag., vol. 8, (1989) September, pp. 263-269.

[2] A. Hoover, V. Kouznetsova and M. Goldbaum, "Locating Blood Vessels in Retinal Images by Piecewise Threshold Probing of a Matched Filter Response", IEEE Transactions on Medical Imaging, vol. 19, no. 3, (2000) March, pp. 203-210.

[3] A. Abdel-Haleim Abdel-Razik Youssif, A. Zaki Ghalwash and A. Ahmed Sabry Abdel-Rahman Ghoneim, "Optic Disc Detection From Normalized Digital Fundus Images by Means of a Vessels' Direction Matched Filter", IEEE Trans. Med Img., vol. 27, no. 1, (2008) January.

[4] B. Cote, W. Hart, M. Goldbaum, P. Kube and M. Nelson, "Classification of blood vessels in ocular fundus images", Computer Science and Engineering Dept., Univ. of California, San Diego, Tech Rep., (1994).

[5] A. Hoover and M. Goldbaum, "Fuzzy convergence", Proceedings of IEEE Conf. Computer Vision and Pattern Recognition, (1998), pp. 716-721.

[6] A. Hoover and M. Goldbaum, "Illumination equalization of a retinal image using the blood vessels as a reference", Proc. Annu. Meeting of the Association for Research in Vision and Ophthalmology (ARVO), (2001).

[7] STARE project website (2003, July): http://www.cecas.clemson.edu/ ahoover/ stare.

[8] A. Hoover and M. Goldbaum, "Locating the optic nerve in a retinal image using the fuzzy convergence of the blood vessels", IEEE Transactions on Medical Imaging, vol. 22, no. 8, (1998) August, pp. 951958.

[9] A. Hoover, G. Jean-Baptiste, X. Jiang, P. J. Flynn, H. Bunke, D. Goldgof, K. Bowyer, D. Eggert, A. Fitzgibbon and R. Fisher, "An experimental comparison of range image segmentation algorithms", IEEE Trans. Pattern Anal. Machine Intell, (1996) July, pp. 673-689.

[10] K. Akita and H. Kuga, "A computer method of understanding ocular fundus images", Pattern Recognition, vol. 15, no. 6, (1982), pp. 431-443.

[11] S. Tamura, Y. Okamoto and K. Yanashima, "Zero-crossing interval correction in tracing eye fundus blood vessels", Pattern Recognit., vol. 21, no. 3, pp. 227-233, (1988).

[12] Y. Sun, "Automated identification of vessel contours in coronary arteriogram by an adaptive tracking 
algorithm”, IEEE Trans. Med. Imag., vol. 8, (1989) March, pp. 78-88.

[13] Y. Tolias and S. Panas, "A fuzzy vessel tracking algorithm for retinal images based on fuzzy clustering”, IEEE Trans. Med. Imag., vol. 17, (1998) April, pp. 263-273.

[14] A. Hoover, G. Jean-Baptiste, D. Goldgof and K. Bowyer, "A methodology for evaluating range image segmentation techniques", Second IEEE Workshop Applications Computer Vision, Sarasota, FL, (1994), pp. 264-27.

[15] W. Kubinger, M. Vincze and M. Ayromlou, "The Role of Gamma Correction in Colour Image Processing", Image Processing, no. 1, (1998), pp. 3-6.

[16] M. Jamshidi, H. Rabbani, Z. Amini, R. Kafieh, A. Ommani and V. Lakshminarayanan, "Automatic Detection of the Optic Disc of the Retina: A Fast Method", J Med Signals Sens, vol. 6, no. 1, (2016).

[17] M. Bhat, M. S. Trun Patil, M. P. Shrinivas and K. S. Geetha, "Automated retinal Optic disc detection using pixel based multi fractal analysis", IEEE International Conference on Computer, Communication and Signal Processing, (2017). 